\title{
Analysis on Problems in Management of Hot Spring Scenic Spot in the North Slope of Changbai Mountain*
}

\author{
Liguang Zhao \\ School of Taxation \\ Jilin University of Finance and Economics \\ Changchun, Jilin, China 130117
}

\author{
Mingju Liu \\ Yatai School of Business \\ Jilin University of Finance and Economics \\ Changchun, China 130117
}

\author{
Xuelian Zheng \\ Yatai School of Business \\ Jilin University of Finance and Economics \\ Changchun, China 130117
}

\begin{abstract}
Located in the southeast of Jilin province, Changbai Mountain is a national forest nature reserve on border line of China and North Korea. It is the key area that the tourism industry gathers in Jilin province. The Changbai Mountain area has picturesque scenery and distinct four seasons. The hot spring tourism is unique. But problems also exist in the development and management. The paper analyzes general situation of hot spring scenic spot in North Slope of Changbai Mountain and problems existing in the management and proposes countermeasures to optimize hot spring scenic spot in North Slope of Changbai Mountain.
\end{abstract}

Keywords-the north scenic area in Changbai Mountain; management of scenic area; product development

\section{General Situation OF Hot SpRING SCENIC SPOT IN NORTH SLOPE OF CHANGBAI MOUNTAIN}

\section{A. Introduction to the North Scenic Area of Changbai Mountain}

Changbai Mountain is the mountain that divides China and North Korea. It is also one of the ten famous mountains in China. Changbai Mountain is rated as national 5A scenic area and famous as "the Most Beautiful Mountain in northeast China". Located in southeast of Jilin province, Changbai Mountain stretches across Antu County in Yanbian Korean Autonomous Prefecture of Jilin province and Fusong County in Baishan city.

The north scenic area of Changbai Mountain has typical volcanic landform. A series of natural scenic spots form here, such as underground forest, Luyuantan Pool, Small Tianchi, hot spring, waterfall and Tianchi. Baiyun Peak, the highest peak of Changbai Mountain has the altitude of 2,691 meters, which is also the highest peak of northeast China. The north scenic area of Changbai Mountain has complete ecological system, complicated natural environment and rich species of

Fund project: National Natural Science Foundation of China (project number: 41401146); Science and Technology Development Project in Jilin Province (201502040NY). plants, which show regular zonal distribution. It shows the unique phenomenon that "a mountain has four seasons and different sceneries because of different weather". It is the main base for experts to research animals and plants.

At present, the development of tourism resources in north scenic area of Changbai Mountain still focuses on closed sightseeing of natural resources.

\section{B. Introduction to Hot Spring Scenic Spot in North Slope of Changbai Mountain}

The hot springs of Changbai Mountain is about 900 meters away from the Changbai Waterfall. There are dozens of springs in the area of more than 1,000 square meters. Boiling water springs out from cracks of the rock continuously, just as dragons spray water. So Changbai Hot Spring is also called Julong Hot Spring. The temperatures of springs are different, from $60{ }^{\circ} \mathrm{C}$ to $82^{\circ} \mathrm{C}$. They are hot springs with high temperature. The Changbai Hot Spring group is the resort with unique scenery. The water in the hot spring is called "magic water", which has high medical value. According to the results of scientific tests, there are dozens of microelements and mineral substances beneficial for human body in the water Bathing in the water of hot spring can make people relaxed and treat many diseases.

\section{CuRRENT Situation OF DeVElopment OF Hot SPRING SCENIC SPOT IN THE NORTH SLOPE OF CHANGBAI MOUNTAIN}

In recent years, the tourism development of Changbai Mountain scenic area centers on Tianchi. The tourism in the north scenic area focuses on the sightseeing of natural scenery. The development of humanistic and cultural tourism resources is not enough. One reason is improper tourism management and corresponding human coordination. Another reason is the 
consideration of environmental protection and natural condition.

At present, the current situation of management of hot spring scenic spot in the North Slope of Changbai Mountain is as follows:

\section{A. Organizational Structure of the Scenic Area}

The north scenic area of Changbai Mountain is in the charge of chief executive of the scenic area. There is general manager in the north scenic area. The scenic areas subordinate to it sets up administration offices. Administration offices are set up in hot spring scenic spots, including six departments, namely information department, marketing department, financial department, security department and auditing department. It also sets up group of centralized management in busy season.

\section{B. Current Situation of Development and Utilization of Hot Spring Resource}

The tourism resources of hot spring in the north scenic area of Changbai Mountain are very rich, among which the natural resources is the most significant. The whole hot spring scenic area is divided into two major parts, including hot spring for sightseeing and hot spring for leisure. In recent years, the hot spring scenic spot of Changbai Mountain focuses on sightseeing.

The hot spring for leisure is to use the advantaged natural resource of hot spring in Changbai Mountain scenic area, develop outdoor hot spring bath pool and provide hot spring bath and hot spring foot bath for tourists. However, according to deep researches, experts of development and management in the scenic area think Changbai Mountain is nature reserve. Setting up hot spring bath in the reserve will cause such pollution problems as sewage discharge and damage resources of natural protection. Therefore, tourists cannot see outdoor hot spring bath in the north scenic area of Changbai Mountain.

In order to let tourists touch hot spring more directly, a pool reserves in east side of the hot spring square for tourists to touch hot spring water closely. Besides, developers of natural resources of the hot spring use pipeline to bring hot spring water in hotels inside and around the scenic area. Tourists can enjoy hot spring bath in hotels with complete facilities. It protects natural resource of hot spring and meets the expectation of tourists to take a bath in hot spring. There are many hot spring hotels around the north scenic area of Changbai Mountain, including Lanjing Hot Spring Resort Hotel, Dazong Dayu Hotel, etc.

The temperature of water in Julong Hot Spring can reach up to 82 degrees. Characteristic food such as egg and corn boiled by hot spring water is popular among tourists, who insist on eating eggs boiled by hot spring water when they visit Changbai Mountain. These eggs are boiled from inside to outside. The yolk is boiled thoroughly but the egg white is not. But it has unique and delicious with smooth taste. Eggs boiled by hot spring water absorb mineral substances in the water, which can supplement many nutrient substances required by people in daily life. It plays the effects of keeping in good heath and heath care.

Because the altitude of Changbai Mountain is high and the mountaintop has accumulated snow all year round, in most time, the temperature of the north scenic area of Changbai Mountain is below zero. Bring natural hot spring water in the pipeline leading to rest area of tourist and administrative staff. It will form natural heating facilities.

\section{Problems In DeVElopMent OF Hot SPRING SCENIC SPOT IN THE NORTH SLOPE OF CHANGBAI MOUNTAIN}

\section{A. Tourism Product of Hot Spring Is Single, Lacking of Image Building.}

Each tourist attraction has unique theme when developing scenic spots. It will accelerate the image building of the scenic spot and let these scenic areas become more attractive. At present, the hot spring scenic spot in the North Slope of Changbai Mountain only has sightseeing. There is only pool for tourists to touch the hot spring water. Most tourism products are limited to bath and recuperate, lacking corresponding derivative products. The hot spring scenic spot in the North Slope of Changbai Mountain lacks tourism image positioning of hot spring. The tourists do not understand hot spring scenic spot. They often ask the staff why the hot spring is so small and they cannot take a bath in hot spring.

\section{B. The System of Management Organization Remains to Be Strengthened.}

The north scenic area of Changbai Mountain now has good formation of management positions. The human resources are also abundant. With gradual development of tourism market, it has higher requirements for professional literacy of administrators and staff of tourism industry. At present, the north scenic area of Changbai Mountain does well in the management of talents. But the government participates in the enterprise management, so that the administration authority is not clear in the hot spring scenic. The scenic spot is divided and managed by different departments. Different departments are under the control of different government sectors. For example, the forest area is managed by the forestry sector. Hot spring area is managed by the department of land and resources. So the government administration is accumulated, which causes problems in the management of scenic spots to some extent.

Moreover, the hot spring scenic spot in the North Slope of Changbai Mountain is adjacent to the waterfall scenic spot. In work handover, the function of departments is unclear. The management of regions is chaotic. The handover of tourists at peak times is not perfect. The authority of department restricts each other. When problems happen, the management system and management mode are backward, which greatly restrict the development and utilization of tourism resources in hot spring scenic spot of the North Slope of Changbai Mountain.

\section{Rich Resources Are Not in Proper Protection}

Changbai Mountain has volcanic geology. The resource of hot spring in the north scenic area of Changbai Mountain is 
extremely rich. But meanwhile the ecological environment is fragile. The tourist facilities in hot spring scenic spot of the North Slope of Changbai Mountain are backward. The management of ecological environment is also improper. Although the hot spring resources are abundant, random exploitation, waste and pollution of hot spring resources happen frequently. It leads to the fact that the quality of water resources in the local area worsens. The natural environment is also damaged to some extent. The fragility of natural resources in the hot spring must be understood clearly. The hot spring resources in the north scenic area of Changbai Mountain are provided for many hot spring hotels in and outside the scenic area. Overexploitation of hot spring water will cause the fall of underground water level and damage the geologic structure. Hot spring resources are polluted and damaged easily. Once the quality of hot spring water changes, it will cause severe damage to hot spring resources. At the meantime, it will cause serious influence on the sustainable development of the hot spring resources.

\section{Infrastructure Projects Are Backward}

At present, the hot spring scenic spot in the North Slope of Changbai Mountain centers on sightseeing. The infrastructure construction is also single and the number is inadequate. The hot spring scenic spot lacks comprehensive management and planning of sustainable development of the scenic spot, as well as corresponding infrastructure and service facility. The main problems include the transport of hot spring water and the disposal of drainage system. The problem of the transport of hot spring water is that because it is a far distance to bring hot spring water in hot spring hotels in and outside the scenic area with the help of pipeline, the water temperature drops, mineral elements lose and the water quality is damaged, so that the effect and medical function reduce; the drainage system in the hot spring area is imperfect. Therefore, in order to protect the natural protection system of Changbai Mountain, some facilities used in bathing pool of hot spring and foot bath of hot spring in the hot spring scenic spot are closed. The hot spring scenic spot lacks attraction. The degree of satisfaction of tourists and the rate that tourists visit again reduce. Meanwhile, the economic benefit of the scenic area drops.

\section{E. The Resource Utilization Pattern Is Largely Identical but with Minor Differences}

Influenced by the natural environment, the hot spring scenic spot in the North Slope of Changbai Mountain does not make market analysis on the management of tourism products. It did not do systematic research on the requirements of tourists. So problems such as the tourism products are the same and the supply does not conform to the demand. The tourism development and utilization of hot spring in Changbai Mountain are largely limited to bath and recuperation and most of them are repeated. They seldom develop comprehensive projects such as leisure, health care, entertainment, popularization of science and sightseeing in hot spring area. It lacks special products for old consumers, business consumers and female consumers. The tourism of hot spring in Changbai Mountain is not in close relationship with medical treatment and the science of health care. Therefore, the motive force of development of tourism resources of hot spring in Changbai Mountain is insufficient and the attraction greatly weakens.

\section{COUNTERMEASURES TO OPTIMIZE HOT SPRING SCENIC SPOT IN THE NORTH SLOPE OF CHANGB AI MOUNTAIN}

The hot spring scenic spot in the North Slope of Changbai Mountain is important natural tourism resources in the Changbai Mountain area. Proper development and utilization of hot spring resources will drive the tourist economy of Jilin province. Therefore, in the construction of scenic spots, we should refer to areas with advanced experience in development and utilization of hot spring resources, even advanced management concept of foreign countries. In order to optimize hot spring scenic spot in Changbai Mountain, we should pay attention to the following aspects:

\section{A. Develop Innovative Products and Strengthen the Building of Tourism Image}

To improve the current situation of hot spring scenic spot in the North Slope of Changbai Mountain, it needs to develop tourism products and image building. Firstly, make market analysis and coordinate the contradiction between market demand and resource protection. Combine with local culture to reasonably develop tourism products of hot spring culture, such as rural home inns of hot spring and activities to experience folk custom of hot spring; secondly, base on the market to boost the publicity and actively participate in travel fair at home and abroad yearly, launch tourist activities and publicize tourist resources and humanistic characteristics of hot spring in the north scenic area of Changbai Mountain; thirdly, comprehensively carry out the publicity of the new media, pack hot spring scenic spot as tourist products, publicize through cooperation with media and internet, properly put it in the publicity and report of media on urban construction of Jilin city to further increase the popularity of hot spring scenic spot in Changbai Mountain and make series of advertising video of Changbai Mountain to make the image of hot spring scenic area more distinctive and attractive.

\section{B. Strengthen Management Organization System}

To improve management level of hot spring scenic spots in the North Slope of Changbai Mountain and establish scientific management pattern, it needs to clearly know the administrative subject, change the chaotic situation of integration of government administration with enterprise and implement centralized and unified professional management of organization in the scenic area and build specialized management organization; secondly, clearly know the property relations of scenic spots, separate the ownership of economic assets from that of the resources of state-owned assets and carry out clear authority separation to avoid the post of management organization in the scenic spot becomes empty or repeats; finally, implement specialized operation of enterprise with clear rights and obligations for the management right of the above organizations. The government conducts macro supervision and adjusts and controls problems such as fund shortage and resource development and deal with the contradiction between resource development and environmental protection. 


\section{Ensure Sustainable Development of Tourist Resources}

Changbai Mountain is national nature reserve, so the development of tourist resources in hot spring scenic area and conservation of natural resources are conflicting. Analyzing from the perspectives of protection and development of tourist resources, it needs to insist on scientific development and ensure the sustainable development of resources of the scenic area. In the development of hot spring resources, social benefit, economic benefit and ecological benefit must be taken into full consideration. In the development of tourist resources, the protection must be put in the first place. Before tourism development, it needs to simulate exploitation scale, bearing capacity of the environment and market tourist source to formulate some measures of environmental protection. Strictly protect resources that are not exploited yet, ensure sustainable development of resources developed to make the hot spring scenic spot continuously get considerable benefit continuously. So it is very necessary to take different measures when protecting resources.

\section{Implement Infrastructure Projects}

Aiming at current situation of infrastructure and service facility of hot spring scenic spot in the North Slope of Changbai Mountain, it needs to transform in the following two aspects. On one hand, adopt high technology and intensify the investment of science and technology, solve problems in the transport of hot spring water and drainage system. After the problems are solved, it can greatly reduce the damage to resources and environment of the scenic area, develop more tourism products of hot spring for tourists to experience and improve service quality of the scenic area, increase appeal and economic income of the scenic spot. On the other hand, pay attention to basic service construction in detail, such as the signboard of the exit and entrance of plank road in Julong Hot Spring, warning board set up in hazardous location of watercourse, signboard of the direction of plank road and rail fence of watercourse. It can protect natural resources, protect tourists and maintain tour order in the scenic area.

\section{E. Strengthen the Utilization of Tourist Resources}

The tourist resources of hot spring are rare in our country. It is the primary task of tourist attraction to make the utilization of tourist resources diversified and reasonable. They can adopt comprehensive development mode to strengthen attraction of the hot spring scenic spot. Firstly, integrate hot spring tourism with science popularization education. Tourists can learn much knowledge related to hot spring in the tour, such as the health care function of microelement contained in hot spring water. They can design interesting tourist activity with knowledge to make tourists learn scientific knowledge in relaxed and interesting environment; secondly, integrate hot spring tour with local cultural experience. Our country has a long history of the culture of hot spring bath. The hot spring scenic spot in Changbai Mountain will have unique and attractive if the culture of hot spring bath of Changbai Mountain is designed through combination of folk culture with living habits in Jilin province; thirdly, integrate hot spring bath with health care. Hot spring water can help people to keep fit through micro mineral elements. It belongs to traditional bath mode. If they use science and technology to assist and add traditional Chinese herbal medicine and precious medicinal materials of Changbai Mountain, research plan to treat diseases and develop rich hot spring products of medical care. Therefore, activities of hot spring bath related to health care such as treatment of disease, preservation of health and body care can be implemented.

\section{CONCLUSION}

Hot springs in north China is more attractive because "snow is falling outside the hot spring; the hot gas in hot spring curls up". It is novel, exciting and challengeable, which will leave a deep impression on people. The resource of hot spring in north scenic area of Changbai Mountain is abundant. People are more inclined to develop and use it. It plays a significant role in the construction of hot spring scenic spot through better grasping the market and the consumption psychology of consumers. However, when developing, we should pay more attention to the fragility of natural environment. It has practical significance on developing the tourist industry of hot spring in north scenic area of Changbai Mountain through finding good development patterns. If the hot spring scenic spot in the North Slope of Changbai Mountain wants to keep a foothold and grow in the tourism market with fierce competition, it should refer to and develop as well as find the road of self innovation.

\section{REFERENCES}

[1] Zhang Yingchao. Analysis on Problems Related to the Management of Scenic Area [J], China Science and Technology Review, 2013, 32 (5): $12-15$

[2] Zou Tongqian. Walk out of the Strange Circle of Scenic Area Management $[\mathrm{J}]$, Tourism World: Tourism Development Research, 2013(4)

[3] Wang Xiaoying. Assumption to Introduce NGO Operation Mechanism in the Management of Scenic Area [J], Economic Research Guide, 2013(21)

[4] An Dong. Comparison and Analysis on Different Types of Management Patterns of Scenic Area [J], Shanxi Science and Technology, 2012(3)

[5] Qiao Shuying, Chen Nan. Analysis on Current Situation of Scenic Areas in Shijiazhuang and Promotion Countermeasures [J], Knowledge Economy, 2013(6)

[6] Jin Xiaozhe. The Management of Scenic Area Should Be Different from That of the "Development Zone" [J], China Economic Weekly, 2013(3) 